\title{
Intermédialités
}

Histoire et théorie des arts, des lettres et des techniques

Intermediality

History and Theory of the Arts, Literature and Technologies

\section{De la critique des dispositifs à l'intermédialité pour approcher les productions artistiques : bilan des travaux du séminaire Intermedialidades (Université Toulouse-Jean Jaurès, France) Exploring art productions, from dispositive theory to intermediality: An overview of the work of the Intermedialidades seminar}

\author{
Fabrice Corrons, Emmanuelle Garnier, Euriell Gobbé-Mévellec, Émilie Lumière, \\ Monique Martinez Thomas, Agatha Mohring, Bruno Péran, Agnès Surbezy et \\ Anne-Claire Yemsi-Paillissé
}

Numéro 30-31, automne 2017, printemps 2018

URI : https://id.erudit.org/iderudit/1049955ar

DOI : https://doi.org/10.7202/1049955ar

Aller au sommaire du numéro

Éditeur(s)

Revue intermédialités (Presses de l’Université de Montréal)

ISSN

1920-3136 (numérique)

Découvrir la revue

Citer cet article

Corrons, F., Garnier, E., Gobbé-Mévellec, E., Lumière, É., Martinez Thomas, M. Mohring, A., Péran, B., Surbezy, A. \& Yemsi-Paillissé, A.-C. (2017). De la critique des dispositifs à l'intermédialité pour approcher les productions artistiques : bilan des travaux du séminaire Intermedialidades (Université Toulouse-Jean Jaurès, France). Intermédialités / Intermediality, (30-31).

https://doi.org/10.7202/1049955ar

\section{Résumé de l'article}

À l'occasion de la publication du numéro "Cartographier/Mapping » de la revue Intermédialités, le groupe des hispanistes du laboratoire LLA-CREATIS de l'Université Toulouse-Jean Jaurès a souhaité établir un bilan des travaux qu'il a menés depuis quatre ans en lien avec l'intermédialité, au sein du séminaire Intermedialidades. L'article s'attache ainsi à dégager les principales lignes de force de ces travaux, notamment : l'articulation des outils de l'intermédialité avec ceux de la critique des dispositifs développée au sein du laboratoire ces dix dernières années; l'intérêt de l'intermédialité entendue comme une approche conceptuelle plurielle pour explorer « l'entre-jeu complexe des médias » (Müller) dans toute production culturelle - artistique ou non -, quelles que soient son ampleur et sa complexité, et en mesurer finement les multiples enjeux; l'hypothèse, enfin, que dans cette perspective très large, il soit possible d'affirmer une spécificité du média artistique, que l'analyse d'objets intermédiaux permet de percevoir dans la mesure où ces objets peuvent porter la trace d'une intention artistique intermédiale, réfléchissant précisément sur la porosité, l'articulation, la friction entre les médias et sur la place de la création dans l'espace médiatique où elle émerge. 


\title{
De la critique des dispositifs à l'intermédialité pour approcher les productions artistiques : bilan des travaux du séminaire Intermedialidades
}

\author{
Fabrice Corrons, Emmanuelle Garnier, \\ Euriell GobBÉ-MÉvellec, Émilie lumière, \\ Monique Martinez Thomas, Agatha Mohring, Bruno \\ PÉran, Agnès Surbezy, Anne-Claire Yemsi-Palllissé \\ LLA-CREATIS \\ (Université Toulouse-Jean Jaurès, France)
}

\section{INTRODUCTION}

l'occasion de la publication $\mathrm{du} \mathrm{n}^{\circ} 29$ de la revue Intermédialités, «Cartographier/Mapping », le groupe des hispanistes du laboratoire LLACREATIS de l'Université Toulouse-Jean Jaurès a souhaité établir un bilan des travaux qu'il a menés depuis quatre ans en lien avec l'intermédialité. Le présent article est le fruit de cette synthèse, que nous avons envisagée comme un espace de « réflexion » dans lequel pouvaient s'exprimer à la fois nos différences de point de vue et des convergences que nous n'avions pas forcément verbalisées, pour nous interroger sur le chemin parcouru durant les premières années d'un séminaire que nous avons baptisé Intermedialidades.

Tout au long de ce séminaire, il semble qu'une réelle continuité s'est construite entre l'intermédialité et la critique des dispositifs développée au sein du laboratoire durant les dix dernières années (École de Toulouse ${ }^{\mathrm{I}}$ ). Pour beaucoup d'entre nous, l'ouverture à l'approche intermédiale a en effet signifié un approfondissement d'outils d'analyse transdisciplinaires, capables d'envisager des productions artistiques hétérogènes et de tenir compte du contexte de leur réception (Partie I).

\footnotetext{
${ }^{\mathrm{I}}$ Ce courant est labellisé par Bernard Vouilloux, dans la revue Critique, comme « l'École de Toulouse » (son lieu de naissance) : «La critique des dispositifs $»$, Critique, $\mathrm{n}^{\circ} 7 \mathrm{I} 8$, mars 2007, p. I52-168.
} 
Le séminaire a d'ailleurs mis en évidence le net intérêt des chercheurs du groupe pour des formes artistiques hybrides (performance, haute gastronomie) ou émergentes (websérie, roman graphique, album numérique pour la jeunesse), sans que nous parvenions à déterminer clairement si ce sont les concepts de l’intermédialité qui nous ont conduits vers ces objets ou bien si la nécessité de trouver des outils adaptés à ces productions nous a menés à l'intermédialité. Certains travaux de l'équipe portent également sur des objets ne relevant pas du champ de l'art (le congrès, le cours de langue étrangère) mais pour lesquels le cadre conceptuel de l'intermédialité (envisager le congrès comme média, explorer le réseau d'interactions médiatiques qui sous-tend le cours de langue, par exemple) nous permet d'explorer la transférabilité et l'opérabilité d'outils d'analyse de situations pensés depuis le champ artistique (Partie 2).

Il était particulièrement intéressant pour notre groupe de disposer d'une approche systémique permettant d'étudier, dans le foisonnement médiatique contemporain, les interactions, les frictions, les convergences entre plusieurs supports de communication et de représentation qui s'offrent à l'utilisateur, dans la mesure où elles nourrissent les productions artistiques. Une telle approche permet de révéler l'insuffisance d'une perspective « insulaire ${ }^{2}$ » centrée sur les médiums artistiques et, à l'inverse, en mettant en lumière la porosité des frontières entre médias de masse et médias artistiques, de déplacer l'analyse vers les modalités et les effets de ces interactions.

Le caractère systémique de cette approche conduit cependant à une forme de déhiérarchisation entre toutes les formes médiatiques. Or, notre groupe, tout en reconnaissant l'importance d'une approche globale, embrassant de manière équivalente toute production humaine ou sociale, s'intéresse principalement - parce que nous parlons depuis un laboratoire dont les arts sont la spécialité —à la spécificité des productions artistiques. Parce que nous n'avons pas souhaité effacer la tension qui se jouait là, nous avons postulé l'existence de « médias artistiques ${ }^{3}$ », c'est-à-dire

\footnotetext{
${ }^{2}$ François Albera, Alain Boillat, Alain Carou et Laurent Le Forestier, « Pour une nouvelle histoire du cinématographe : Cinq questions à André Gaudreault $\gg, 1895$, Mille buit cent quatre-vingt-quinze, Revue de l'association française de recherche sur l'bistoire du cinéma, n ${ }^{\circ} 57$, 2009, p. I2, http://journals.openedition.org/1895/4005 (consultation le 6 décembre 2017).

3 Devant la tension conceptuelle entre « medium » et « média », nous avons décidé de privilégier la terminologie « média » puisque nos recherches portent sur les relations intermédiales et non sur l'essentialisation du médium, Éric Méchoulan, « Intermédialité, ou comment penser les transmissions », Fabula/Les colloques. Création, intermédialité,
} 
de supports capables d'établir avec et entre des sujets une communication que l'on peut interpréter en termes d'expérience esthétique, et où se manifeste l'expression, l'intention d'un ou plusieurs artistes. Ainsi, il nous a semblé qu'on pouvait observer l'existence d'objets intermédiaux ${ }^{4}$, c'est-à-dire portant la trace d'une pensée artistique intermédiale, réfléchissant sur la porosité, l’articulation, la friction entre les médias et sur la place de la création dans l'espace médiatique où elle émerge (Partie 3). Notre particularité dans le paysage des études intermédiales serait donc peut-être à situer dans la réaffirmation de la spécificité de l'artistique et du propos de l'artiste. Il s'agit là à la fois d'un point de convergence de nos travaux à l'heure actuelle, et d'une piste en cours d'exploration, présentée à la fin de cet article mais qu'il s'agira de théoriser de façon solide dans une prochaine étape.

\section{MIGRER / MIGRAR: DE LA CRITIQUE DES DISPOSITIFS À L'INTERMÉDIALITÉ}

L'équipe toulousaine a rencontré le concept d'intermédialité comme une sorte d'évidence épistémologique: au terme d'une quinzaine d'années de développement de la critique des dispositifs, une partie des chercheurs qui avaient participé à l'élaboration de cette théorie (Marie-Thérèse Mathet, Stéphane Lojkine, Philippe Ortel, Arnaud Rykner, etc. ${ }^{5}$ ) se sont naturellement interrogés sur les liens

dispositif, 5 mars 2017, http://www.fabula.org/colloques/document4278.php (consultation le 3I mai 2017).

$4 \mathrm{Au}$ fur et à mesure de nos travaux, il nous est apparu nécessaire de distinguer entre le caractère plurimédiatique (ou multimodal) d'un objet où coexistent plusieurs médias, et son caractère intermédiatique, lorsque ces médias interagissent entre eux. Nous avons réservé l'adjectif intermédial pour qualifier à la fois notre approche des phénomènes médiatiques et intermédiatiques et les créations artistiques dotées d'une dimension réflexive quant à leur propre nature médiatique. Nous aurons l'occasion de revenir plus loin sur cette question.

${ }^{5}$ La Scène. Littérature et arts visuels, Marie-Thérèse Mathet (dir.), Paris, L'Harmattan, 20oI; L'Écran de la représentation, Stéphane Lojkine(dir.), Paris, L'Harmattan, coll. « Champs visuels », 200I; L'Incompréhensible. Littérature, réel, visuel, Marie-Thérèse Mathet (dir.), Paris, L'Harmattan, coll. « Champs visuels », 2003; Brutalité et représentation, Marie-Thérèse Mathet (dir.), Paris, L’Harmattan, coll. « Champs visuels », 2006; et Discours, Images, Dispositifs, Philippe Ortel (dir.), Paris, L'Harmattan, coll. « Champs visuels », 2008. Ce dernier ouvrage constitue lui-même le second volet d'un diptyque Penser la représentation $I$ et $I I$, en collaboration avec le Centre Joseph Hanse de l'Université de Louvain-La Neuve, dont le premier volet s'intitule : La Littérature à l'ère de la reproductibilité technique. Réponses littéraires aux nouveaux dispositifs représentatifs créés par les médias modernes, Pierre Piret (dir.), Paris, L’Harmattan, coll. « Champs visuels», 2007. La notion s'y élabore 
entre les formes artistiques et les médias de communication. Dans la mouvance de la pensée de Michel Foucault ${ }^{6}$, le dispositif artistique est vu comme un réseau de moyens hétérogènes agencés de façon à produire, dans un espace-temps donné, des effets de sens sur le récepteur. Il est constitué de trois niveaux articulés : (I) le niveau géométral ou technique (la disposition des éléments organisés dans l'espace par la fiction); (II) le niveau pragmatique ou scopique (l'interaction de plusieurs actants sous le regard du spectateur); (III) le niveau symbolique (valeurs sémantiques et axiologiques associées à l'organisation spatiale ${ }^{7}$ ). Le dispositif artistique comportant des éléments hétérogènes (au-delà du rapport texte-image qui était à l'origine de cette nouvelle approche), la diversité du matériau qu'il était susceptible d'agencer a naturellement conduit les chercheurs à s'interroger sur la présence des médias de communication dans les œuvres, sur le statut médiatique de l'œuvre, et sur les relations entre eux.

C’est ainsi qu'en 2013, Philippe Ortel, Monique Martinez et Mireille Raynal lançaient, au sein du laboratoire LLA-CREATIS, un séminaire ${ }^{8}$ visant à préparer le croisement entre les deux concepts et l'enrichissement de la notion de dispositif. À cette époque, ils avaient recours à la notion d'intermédialité, car celle-ci paraissait particulièrement féconde pour approcher des productions artistiques qui intègrent plusieurs médias, artistiques ou non. Cette approche, développée par Monique Martinez Thomas dans « Dispositive, Intermediality and Society: Tales of the Bed in

progressivement, avec une précision et une pertinence toujours accrues, générant de nombreux travaux de thèse. Notre groupe d'hispanistes a défriché les corpus en langue espagnole depuis cette approche à partir de 2007, Monique Martinez Thomas et Euriell Gobbé-Mévellec (dir.) : Dispositivo y artes: una nueva herramienta critica para analizar las producciones contemporáneas (e-book), Ciudad Real, Naque, 2014, www.naque.es/virtuemart/213/I/novedades/dispositivo-y-artes-detail

${ }^{6}$ Michel Foucault, « Le jeu de Michel Foucault », Dits et écrits [1977], tome II, Paris, Gallimard, 1994 p. $298-329$.

7 Cette définition synthétique du dispositif est celle que nous avons proposée en introduction de l'ouvrage Dispositivo y artes: una nueva herramienta crítica para analizar las producciones contemporáneas, Martinez Thomas et Gobbé-Mévellec, 20I4, p. 7. Pour l'articulation avec le dispositif selon Foucault, nous renvoyons à l'article d'Arnaud Rykner dans le même ouvrage: «Acercarse al dispositivo: continuum, interacciones y lógica creadora », p. 13-29.

8 «Séminaire Dispositifs plastiques et intermédialité »: http://lla-creatis.univtlse2.fr/accueil/manifestations-scientifiques/seminaire-dispositifs-plastiques-etintermedialite-I98767.kjsp?RH=I270I25529372 (consultation le 9 juin 20I7). 
Contemporary Spain ${ }^{9} \gg$, permettait un regard nouveau sur l'agencement des différents éléments constitutifs de l'œuvre : il ne s'agissait plus d'étudier les unes à côté des autres des formes de représentation « codifiées », inscrites dans une histoire des genres et correspondant à des horizons d'attente différents, mais d'analyser comment leurs interactions - convergences, frictions, porosités - généraient des écosystèmes chaque fois renouvelés, uniques, rassemblant des utilisateurs différenciés. Ces interrogations encore largement ouvertes n'étaient pas sans incidence sur l'épistémologie de l'art car à partir du moment où les médias, technologiques ou non (texte, peinture, photographie, gravure, lithographie, télévision, vidéo, cinéma, sculpture, numérisation, publicité, etc.) devenaient matériau artistique, la valeur symbolique de l'art était questionnée. Loin de la représentation sacralisée de la figure de l'artiste, loin de la fascination de l'œuvre soumise au regard distancié du récepteur, l'interprétation intermédiale permettait de s'interroger sur les nouveaux modes de médiation, l'émergence de propositions artistiques alternatives, sur la notion d'auctorialité en pleine mutation, sur les processus cognitifs d'une perception souvent rhizomatique.

La seconde étape vers l'intermédialité a été le colloque international Création, intermédialité, dispositif, organisé en 2014 à Toulouse, et dans lequel sont également intervenus des chercheurs du CRIalt ${ }^{\text {Io }}$. Philippe Ortel réaffirmait le lien entre dispositifs et médias dans le programme de cette rencontre :

Au-delà de la coupure - le dispositif est justement ce dépassement de la clôture, puisqu'il sépare et maintient séparé tout en unissant et rapprochant - , coupure des champs disciplinaires, des genres, des pratiques, des modes de communication, il s'agira d'évaluer l'influence réciproque des médiums, l'influence des médiums sur la représentation - le médium

\footnotetext{
${ }_{9}$ Monique Martinez Thomas, « Dispositive, Intermediality and Society: Tales of the Bed in Contemporary Spain », dans Éric Méchoulan (dir.), Intermedialities, SubStance, vol. 44, $\mathrm{n}^{\circ}$ 3, 2015, p. 98-III.

10 Fabula/Les colloques. Création, intermédialité, dispositif, mars 2017, www.fabula.org/colloques/index.php?id=4I54 (consultation le 6 juin 2017). Les captations des interventions sont disponibles à l'adresse suivante: www.canalu.tv/producteurs/universite_toulouse_ii_le_mirail/colloques/creation_intermedialite_dispo sitif (consultation le I7 avril 20I7).
} 
nouveau crée une représentation nouvelle de la réalité —, sur l'imaginaire, sur la pensée, sur la façon de voir, de sentir et de vivre ${ }^{11}$.

Dans la lignée de ces travaux, plusieurs chercheuses ont approfondi, à partir de 20I4, l'articulation entre dispositif et intermédialité ${ }^{12}$. Envisageant le dispositif depuis l'intermédialité, elles montraient que le caractère plurimédiatique et intermédiatique des productions artistiques permettait de porter plus d'attention à la matérialité des éléments agencés dans la forme dispositive (leur support et leur spécificité dans l'agencement de l'espace-temps). Elles dépassaient ainsi, grâce à la notion de milieu ${ }^{13}$, le concept de l'individuation de la réception, élément clé de la critique des dispositifs (notamment par le concept d'《autre scène » repris et développé par Stéphane Lojkine $\left.{ }^{\mathrm{I}}\right)$. La prise en compte du milieu recentrait l'analyse sur les acteurs sociaux et leurs échanges avec les autres membres du groupe créé par le média artistique. Des usagers de différentes communautés réunis par les œuvres intermédiatiques étaient susceptibles de se constituer en nouveau(x) milieu(x) doté(s) de problématiques

II Colloque Création, intermédialité, dispositif, I2-I4 février 2017, Université de Toulouse II — Le Mirail: $\quad$ http://lla-creatis.univ-tlse2.fr/accueil/manifestationsscientifiques/creation-intermedialite-dispositif-290333.kjsp?RH=I270I25529372 (consultation le II mars 20I8).

${ }^{12}$ Notamment, Monique Martinez Thomas, à partir d'un corpus cinématographique : «L'impératif du vivant: l'intermédialité face à la conscience. Medianeras de Gustavo Taretto », Fabula/Les colloques, Création, intermédialité, dispositif, mars 2017, www.fabula.org/colloques/document4469.php (consultation le II mars 2018). et « Dispositive, Intermediality and Society: Tales of the Bed in Contemporary Spain », Éric Méchoulan(dir.), Intermedialities, SubStance, vol. 44, $\mathrm{n}^{\circ}$ 3, 20I5, p. 98-III; Emmanuelle Garnier : «El dispositivo intermedial de Metamorfosis: una reescritura escénica de la novela de Kafka por La Fura dels Baus », dans Amélie Florenchie et Dominique Breton (dir.), Nuevos dispositivos enunciativos en la era intermedial, Villeurbanne, Orbis Tertius, 2015, p. 89-107; et «Théâtre performatif et dispositif: une approche de Todo el cielo sobre la tierra (El síndrome de Wendy) de Angélica Liddell », participation au colloque « Texte et performance : au croisement des arts visuels et des arts du spectacle », I-3 octobre 20I4, Université de Strasbourg, actes à paraître.

${ }^{13}$ « A medium is not defined solely by its tangible support or means, but also by social actors. The user at the heart of the mediatized dispositive is central, as are users' exchanges with others members of the group created by the medium», dans Monique Martinez Thomas, «Dispositive, Intermediality and Society: Tales of the bed in Contemporary Spain », dans Méchoulan, Éric (dir.), Intermedialities, SubStance, vol. 44, n³ 3, 2015, p. IOI.

${ }^{14}$ Stéphane Lojkine, La Scène de roman: méthode d'analyse, Paris, Armand Colin, coll. « », 2002. 
sociétales ou historiques partagées. L'intermédialité permettait alors de renouer avec le politique, souvent évacué par la critique des dispositifs. recentrait son travail sur l'intermédialité en ouvrant le séminaire Intermedialidades et en accueillant plusieurs chercheurs spécialistes de l'intermédialitérs. Au-delà de la perception de l'intermédialité comme phénomène, l'équipe a également orienté sa réflexion sur l'intermédialité comme approche, comme regard. La pertinence de l'intermédialité en tant qu'approche tient à sa capacité à ouvrir des théories déjà novatrices ou classiques à la prise en compte de la matérialité et du milieu des productions, ce qui permet de mieux appréhender des objets construits sur un maillage médiatique mouvant et donc difficilement saisissable (le mythe en est un exemple $\left.{ }^{16}\right)$. Si l'on considère que notre conception de toute « réalité », présente ou ancienne, passe par des mécanismes d'interprétation et de représentation, c'est-à-dire par des processus de médiation, le regard intermédial nous offre une acuité toute particulière, alliée en outre à une grande liberté théorique. Et c'est peut-être là l'une des principales forces de l'approche intermédiale, capable, par la souplesse et la

is Jean-Marc Larrue (CRIalt, Université de Montréal), conférence « Théâtre et intermédialité », 20 octobre 20I4, Université Toulouse-Jean Jaurès ; Philippe Despoix (CRIalt, Université de Montréal), conférence «Approches intermédiales: histoire et contexte canadiens 》, 2 juillet 20I4, Université Toulouse-Jean Jaurès. Rémy Besson (postdoctorant au CRIalt) a notamment rédigé, au sein du laboratoire LLA-CREATIS, pour le LabCom RiMeC, la synthèse «Prolégomènes pour une définition de l'intermédialité à l'époque contemporaine », 20I4, https://hal-univ-tlse2.archives-ouvertes.fr/hal-oroI2325 (consultation le 5 mai 2017), ainsi que les rapports de recherche « Le Congrès comme média. Retour problématisé sur l'observation d'EuroPCR 20I4 》 (2014) et « Une recherche intermédiale mise à l'épreuve du terrain. Retour sur la conception d'une session dite innovante », 20I5. Claudia Polledri, également postdoctorante au CRIalt, a réalisé en 2016, au sein du laboratoire LLACREATIS, pour le LabCom RiMeC, le rapport de recherche « Retransmission et congrès. Étude intermédiale de la session LIVE »; elle a également présenté une analyse de l'œuvre documentaire A Journey de l'artiste libanaise Lamia Joreige dans le cadre du séminaire Intermedialidades (I2 avril 2016, Université Toulouse-Jean Jaurès) ainsi que la communication «Travelling sur les frontières: enjeux de la construction d'un regard sur et au MoyenOrient », dans le cadre du grand séminaire MÉTA\# 2 du laboratoire LLA-CREATIS, « Saisir le mouvement : Enjeux de l'art et de la recherche en arts », Io juin 20I6, Université ToulouseJean Jaurès.

${ }^{16}$ Émilie Lumière, « Intermédialité et mise en scène de l'histoire au théâtre : Retrato de gran almirante con perros [Portrait de Grand Amiral aux chiens] de Luis Riaza », pour la publication L'intermédialité à la croisée des chemins: bilans et perspectives, Peter Lang, à paraître. 
transférabilité de ses concepts, de rassembler des chercheurs issus de domaines différents.

Ces trois années de réflexion collective, fondées sur l'herméneutique de textes théoriques ${ }^{17}$ mais aussi sur l'appropriation des concepts appliqués à nos corpus de recherche ${ }^{18}$, croisés à d'autres théories, ont soulevé de nombreuses questions autour de l'étendue sémantique des termes essentiels que sont l'intermédialité, le média et le médium. De là ont émergé des nuances terminologiques comme la distinction entre « intermédiatique » et « intermédial », concomitantes de notre hypothèse selon laquelle il existerait des objets non seulement intermédiatiques mais également « intermédiaux »- portant la trace d'une réflexion sur les médias et les phénomènes de médiation - et ce tout particulièrement dans le champ singulier de l'art.

\section{EXPLORER / EXPLORAR : L'INTERMÉdiALITÉ, POUR QUELS CORPUS?}

À l'heure de dresser un bilan de nos recherches et d'essayer de repérer des lignes convergentes, force est de constater que la question de l'art a sous-tendu tous nos travaux et que l'intermédialité a joué comme une sorte d'aiguillon, à la fois irritant, en ce qu'il bousculait des définitions établies et des certitudes collectives, et stimulant, en ce qu'il nous a poussés à dépasser nos « zones de confort » habituelles, à approcher des objets plus énigmatiques ou plus foisonnants, plus inattendus, pour lesquels, pourtant, notre point de vue de chercheurs en arts peut s'avérer intéressant.

Nous nous sommes ainsi souvent tournés, durant ces trois années, vers des productions artistiques contemporaines conjuguant une pluralité de modes de représentation et de communication (performances, films, pièces de théâtre, etc.), certains relevant de disciplines artistiques, d'autres non. L'approche intermédiale nous a fourni un cadre de réflexion approprié pour embrasser ces productions plurielles, pour penser les interactions entre différents modes de représentation et de communication en coprésence ainsi que la façon dont ces productions se relient à

${ }^{17}$ Synthèses des travaux d'Éric Méchoulan, André Gaudreault, David Bolter et Richard Grusin, Jürgen E. Müller, Silvestra Mariniello, Henry Jenkins, Jean-Marc Larrue, Simon Hagemann, Marie-Christine Lesage. Une partie de ces notes de lecture a été mise en ligne par Rémy Besson sur le site Cinémadoc: https://cinemadoc.hypotheses.org/3457 (consultation le 4 mai 2017).

${ }^{18}$ Théâtre espagnol contemporain, textes et spectacles; théâtre de rue; arts performatifs; album imprimé et livre numérique pour enfant ; cinéma; websérie; gastronomie; roman graphique; traduction; théâtre appliqué; congrès; didactique des langues. 
leurs destinataires. L'hétérogénéité de ces productions, conjuguée à un cadre conceptuel élargi, nous a par ailleurs conduits à ouvrir notre approche disciplinaire, de façon à étudier des productions ne relevant pas des champs traditionnels des arts, comme la haute gastronomie, par exemple.

Le cadre de l'intermédialité nous a également poussés à étudier de plus près certaines formes médiatiques émergentes dans le champ des arts : websérie, roman graphique, album numérique pour la jeunesse, certains dispositifs théâtraux de rue, etc. Si le caractère très confidentiel, très expérimental de certaines de ces productions dans l'ère hispanique n'a pas constitué pour nous une limite à leur pertinence comme objet d'étude, c'est très certainement parce que les travaux d'André Gaudreault, de Philippe Marion, de Rick Altman, etc. nous ont fourni des cadres de pensée à l'aune desquels évaluer le degré d'émergence de ces formes innovantes.

Parallèlement à ces travaux, nous avons également commencé à explorer des sphères étrangères à nos disciplines, ne relevant pas de l'art, afin de voir si nos outils d'analyse (ceux issus de la critique des dispositifs et ceux de l'intermédialité, notamment) s'avéraient opératoires transposés à d'autres situations (le congrès, le cours de langue étrangère, par exemple). Ces pistes de recherche plus applicatives, en cours, s'avèrent extrêmement stimulantes et nécessiteront dans une étape ultérieure un travail réflexif de l'équipe pour voir si, de leur côté, ces situations, ces objets inédits ont transformé notre approche.

\section{I. L'INTERmédialité POUR APPROCHER DES PRODUCTIONS HÉTÉROGÈNES}

Les concepts de l'intermédialité se sont révélés opératoires pour aborder des formes artistiques hybrides, issues d'une culture contemporaine résolument interactive et de processus de création et de réception qui ont recours, de façon croissante, aux nouvelles technologies et au numérique. Nous retiendrons pour en témoigner l'exemple des créations de «théâtre performance » de la compagnie catalane Agrupación Señor Serrano ainsi que le « dîner performance » intitulé El Somni de Franc Aleu et Joan, Josep et Jordi Roca (20I4).

Les arts de la scène contemporaine multiplient les productions artistiques plurimédiatiques qui impliquent de plus en plus le numérique et qui questionnent souvent le genre théâtral dans sa spécificité, sa supposée « essence ». La compagnie catalane Agrupación Señor Serrano propose ainsi des spectacles qui oscillent entre la performance et un théâtre d'objets connecté aux nouvelles technologies. C'est le cas 
de $A$ House in Asia (2014) ${ }^{19}$ qui dépeint au vitriol notre société mondialisée à travers trois histoires enchevêtrées: l'entêtement des forces spéciales états-uniennes à neutraliser Ben Laden, l'obsession du capitaine Achab à capturer Moby Dick et la guerre contre Geronimo, chef des Apaches, menée par le $7^{\mathrm{e}}$ régiment de cavalerie. L'analyse intermédiale met l'accent à la fois sur l'hétérogénéité médiatique et surtout sur l'interaction inédite et fructueuse entre les nombreux médias convoqués sur scène : figurines, maquettes de maison ou d'avion, table de jeu de société, performersmanipulateurs, danseurs, bande-son, caméras, ordinateur, table de mixage, grand écran à l'arrière-scène. La notion de personnage semble ne pas résister à cette dispersion, à cette atomisation médiatique. Le cowboy, par exemple, s'incarne de différentes façons: personnage physiquement présent sur scène, figurines (en plastique ou de plomb), performer affublé du chapeau typique, danseurs country et, à l'écran, figurines filmées en direct, personnages d'extraits vidéo de western. Il fonctionne comme un révélateur médiatique, obligeant le spectateur à s'intéresser à la matérialité des différentes formes de représentation et aux relations, parfois conflictuelles, qu'elles entretiennent entre elles.

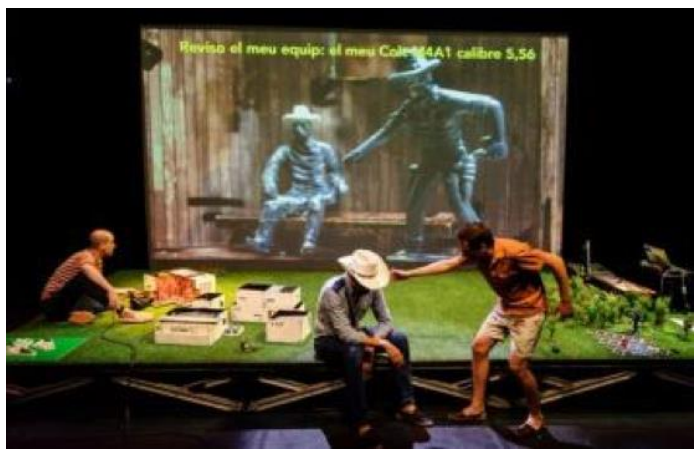

Figure r. L'exemple du cowboy dans $A$ House in Asia, Agrupación Señor Serrano, 2014, photographe : Nacho Gómez)2o.

${ }^{19}$ Le lecteur pourra consulter la page web de la compagnie www.srserrano.com/es/ (consultation le 5 avril 20I7).

${ }^{20}$ L'image est disponible en suivant le lien https://voltarivoltar.com/2015/05/ri/teatre-ahouse-in-asia-\%Fo\% ${ }_{9} \mathrm{~F} \%{ }_{9} 0 \% 8 \mathrm{C} \% \mathrm{Fo} \%{ }_{9} \mathrm{~F} \%{ }_{9} 0 \% 8 \mathrm{C} \% \mathrm{Fo} \%{ }_{9} \mathrm{~F} \%{ }_{9} \% \% 8 \mathrm{C} \% \mathrm{Fo} \%{ }_{9} \mathrm{~F} \%{ }_{9} 0 \% 8 \mathrm{C}$ teatre-auditori-de-granollers/ (consultation le 7 avril 20I7). 


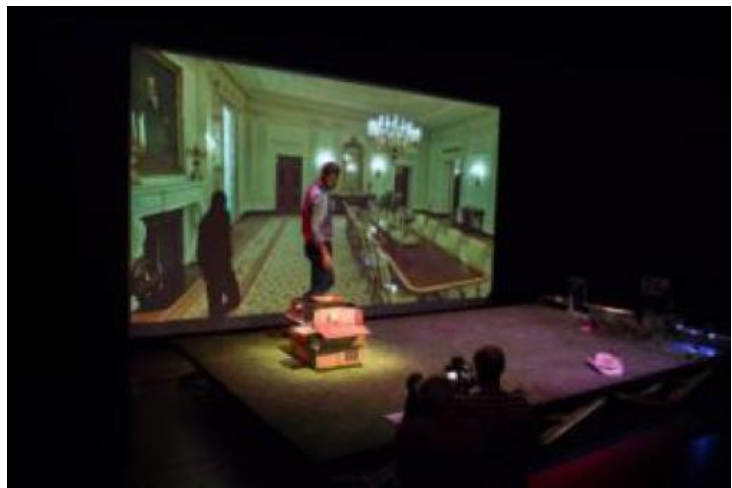

Figure 2. Maquette et projection dans A House in Asia, Agrupación Señor Serrano, 2014, photographe : Nacho Gómez² .

De la même façon, l'autonomie de l'image et du son ou du texte projeté renforce cette hypermédiatisation, cette exhibition de la médiativité : la danse country se fait sur une chanson pop du boysband «Take That»; les sous-titres / surtitres ne correspondent pas toujours à l'enregistrement sonore dans les vidéos avec les Indiens, etc. La profusion médiatique multiplie la combinaison de ces échanges problématiques entre les médias.

L'écosystème médiatique, en ce qu'il favorise ces phénomènes de disjonction entre les médias, facilite la perception du spectateur pour ce qui est des croisements des trois histoires. Autrement dit, c'est parce que les médias, ne cessant de s'entrechoquer, révèlent leur instabilité, leur relativité ou leur interchangeabilité, que l'on peut plus aisément accepter que « l'Indien » soit équivalent au cachalot Moby Dick et que l'antre du monstre marin n'est autre que la maison de Ben Laden, dont on voit la maquette sur scène et la projection à l'écran.

À son tour, la version écranique de la maison de Ben Laden se transforme, au contact de médias hétérogènes (sons, textes, images) intégrés par le technicien de la table de mixage, en un salon de la Maison-Blanche. Ce jeu constant sur les réemplois ou recyclages des médias et sur les multiples identités des référents renvoie à la complexité de notre monde surmédiatisé et déconstruit l'apparente transparence médiatique (immediacy).

En exhibant sur le plateau l'hétérogénéité des « ficelles » de la construction de ce récit médiatique pluriel, $A$ House in Asia souligne l'opacité des médias, éveillant

2I L'image est disponible en suivant le lien www.srserrano.com/ca/a-house-inasia/(consultation le 23 mai 2017). 
chez le spectateur une conscience intermédiale. Qu'en est-il de l'homme dans ce theatrum mundi de bric et de broc ? Il y est représenté - et c'est là la gageure du spectacle et un des apports majeurs de la perspective intermédiale - comme un média à part entière : placé au même titre que les figurines ou la bande-son bigarrée au cœur du maillage intermédiatique, il agit en permanence sur la construction de cette réalité, manipulant et se faisant manipuler par les autres médias. L'intermédialité permet ainsi non seulement d'aborder de manière singulière de telles propositions artistiques difficilement catégorisables mais aussi de mieux appréhender la vision du monde qu'elles véhiculent.

Les études intermédiales se révèlent tout aussi pertinentes pour étudier des formes d'art culinaire d'avant-garde, par exemple les créations de la cuisine « technoémotionnelle ${ }^{22}$ » du début du $2 \mathrm{I}^{\mathrm{e}}$ siècle en Catalogne. L'intermédialité et notamment les concepts de remédiation ${ }^{23}$, de médiativité ${ }^{24}$ et de transmédialité ${ }^{25}$ permettent de saisir non seulement la complexité de l'entrelacs de médias qui constitue ces productions, mais aussi l'inscription plus large de ces dernières dans le maillage médiatique contemporain.

Prenons l'exemple du dîner-performance El Somni (« le rêve », en catalan), créé en 2014 par les frères Roca - les trois chefs cuisiniers du restaurant El Celler de Can Roca à Gérone - et le vidéaste catalan Franc Aleu. Le cœur de ce projet est un dîner gastronomique immersif, qui intègre une grande variété de médias, anciens et nouveaux. Ce dîner, donné au printemps 2014 à Barcelone, au Centre d'art Santa

${ }^{22}$ C'est par ce néologisme que le journaliste gastronomique espagnol Pau Arenos désigne la cuisine de Ferran Adrià et de ses héritiers. Il définit le mouvement technoémotionnel ainsi : «Mouvement culinaire mondial du début du $2 \mathrm{I}^{\mathrm{e}}$ siècle né à El Bulli [...]. L'objectif des plats qui sont servis est de susciter l'émotion du convive, et, pour ce faire, de nouveaux concepts, techniques et technologies sont employés. [...] Les cuisiniers prêtent attention aux cinq sens et pas seulement au goût et à l'odorat. En plus de créer de nouveaux plats, le but est d'ouvrir de nouveaux chemins [...]. Pour accéder à de nouveaux savoirs, les chefs ont établi un dialogue avec les scientifiques, mais aussi avec des artistes, des architectes, des dramaturges, des romanciers, des musiciens, des cavistes, des artisans, des parfumeurs, des poètes, des journalistes, des historiens, des anthropologues, des philosophes, des designers... », Pau Arenos, La cocina de los valientes, Barcelona, Ediciones B, 20Ir. (Notre traduction.)

${ }_{23}$ Jay David Bolter et Richard Grusin, Remediation, Understanding New Media, Cambridge, MIT Press, 1999.

${ }^{24}$ La médiativité est, selon Philippe Marion, « le potentiel expressif et communicationnel développé par le média », Philippe Marion, « Narratologie médiatique et médiagénie des récits », Recherches en communication, $\mathrm{n}^{\circ} 7,1997, \mathrm{p} .80$.

${ }^{25}$ Nous renvoyons au concept tel qu'il est développé par Henry Jenkins, La culture de la convergence, Des médias au transmédia [2006], trad. de l'anglais par C. Jaquet, Paris, A. Colin/Ina Éd., coll. « Médiacultures », 20I3. 
Mònica, rassemble douze personnes, les hôtes, autour d'une table ronde à la surface de laquelle un grand écran est greffé; derrière eux et tout autour, des écrans courbes et des haut-parleurs plongent les mangeurs-spectateurs dans un univers fantasmatique et polysensoriel complexe qui leur raconte - avec des emprunts clairs à l'opéra - une histoire tragique d'amour et de haine, avec des plats, des vins, des musiques et des images créés pour l'occasion.

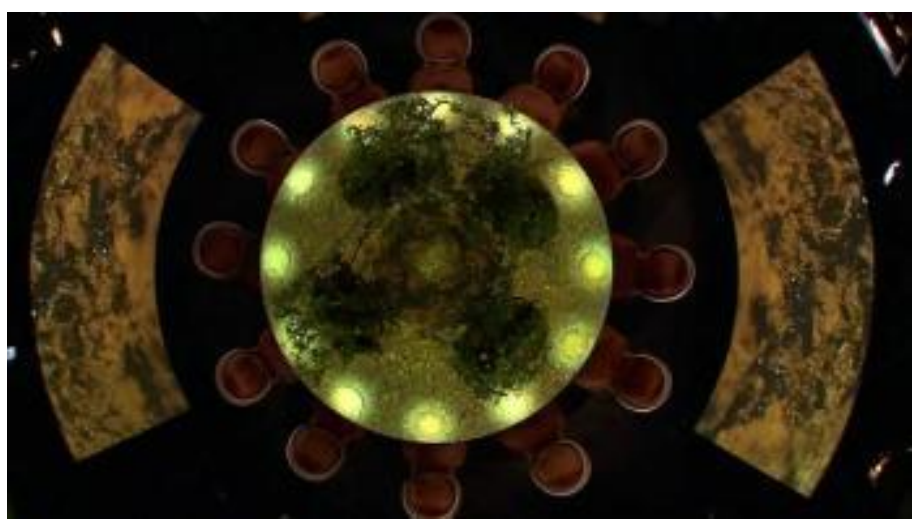

Figure 3. Le dispositif du dîner performance El Somni, les frères Roca, 20I4, photographe anonyme ${ }^{26}$.

I23 Dans cette expérience, on note la coprésence de cinq médias anciens et nouveaux : au restaurant gastronomique - le média englobant, que l'on choisit ici d'appeler le « média hôte » - s'agrègent, invités par le premier, le video art, la musique symphonique, le chant, le design. Le média culinaire se remédie ainsi au contact des autres. Quelle est la conséquence de cette remédiation ? Dans le cas d'El Somni, les convives ont confié que la superposition de signes et de stimuli, si elle a effectivement engendré des émotions fortes, a aussi profondément modifié la dégustation et les échanges. Il semble que, lorsque le potentiel expressif et communicationnel des médias intégrés à la performance culinaire est poussé à son maximum, la médiativité propre au culinaire, avec ses composantes essentielles - la commensalité et le goût -, entre en mutation au bénéfice de l'expérience synesthésique d'un spectacle médiatiquement hybride.

\footnotetext{
26 L'image est disponible en suivant le lien https://i.ytimg.com/vi/foqVFGASzvU/maxresdefault.jpg (consultation le I2 mai 20I7).
} 
L'approche intermédiale permet aussi de prendre en compte les deux niveaux majeurs de la réception, l'individuel et le collectif, à travers la mise en relation de communautés et de milieux situés à la charnière des différents médias, dans l'« intermédias ». Outre le dîner immersif, le projet El Somni comprend un film, un site internet ${ }^{27}$, de la publicité sous diverses formes (trailers, affiches, etc.) et, enfin, un grand livre papier ${ }^{28}$ à mi-chemin entre le livre d'art et le catalogue d'exposition. Cette diversité de médias édifie un véritable univers (assimilable au « world making » de Henry Jenkins ${ }^{29}$ ) et permet à des publics très variés d'accéder à l'expérience - unique et élitiste - du dîner-performance. El Somni est en cela un objet transmédiatique et esthétique complexe, qui fait le pari du mariage « d'amour et de raison ${ }^{30} »$ de la création artistique et de la communication.

L'hybridité est également au cour de l'émergence de médias nouveaux qui se construisent à partir, contre ou avec des médias existants. Par le croisement inédit de genres, ils renouvellent les formes ou en inventent d'autres, qui s'institutionnalisent peu à peu comme des séries culturelles à part entière. Deux de ces médias émergents ont particulièrement retenu notre attention dans la création contemporaine espagnole : le roman graphique et la websérie.

\section{2 L'INTERMÉdIALITÉ POUR ABORDER DES MÉdIAS «ÉMERGENTS ": ROMAN GRAPHIQUE ET WEBSÉRIE}

En constante mutation, exploration et expérimentation, roman graphique et websérie ${ }^{3 \mathrm{I}}$ connaissent dans les années 2000 un tournant définitoire et identitaire :

${ }^{27}$ Le lecteur pourra consulter la page web du projet www.elsomni.cat/es/ (consultation le 6 mai 20I7).

${ }^{28}$ Franc Aleu, El celler de Can Roca, El Somni, Madrid, Lunwerg, 2014.

${ }^{29}$ Jenkins, 2013.

${ }^{30}$ Nous reprenons ici le titre de l'ouvrage d'Alexandre Kson, Art et communication: un mariage d'amour et de raison, Bluffy, Éditions Kawa, 2014.

${ }^{31}$ En nous appuyant sur la définition d'André Gaudreault et de Philippe Marion, pour qui « un média peut [...] se comprendre comme une fédération provisoirement stabilisée de différents paramètres, dont la vocation de circulation transmédiatique s'inscrit dans une perspective plus intermédiale que strictement médiale », André Gaudreault, Philippe Marion, Cinéma et généalogie des médias. Médiamorphoses, Bry-sur-Marne: Institut national de l'audiovisuel, n ${ }^{\circ}$, Dossier : D'un média.... l'autre, 2006, p. 25, nous avons fait le choix de considérer la websérie comme un média et non comme un simple format ou genre. Fédération en cours de stabilisation de différents médias (Internet et la télévision, principalement, mais aussi la publicité, le cinéma, etc.), la websérie est non seulement issue de processus de 
médias en cours de sérialisation, ils se développent et cherchent à se définir essentiellement dans les relations qu'ils établissent, ou rejettent, avec d'autres médias.

Depuis cette date, le roman graphique espagnol affirme sa différence par rapport à la bande dessinée, ses spécificités artistiques ou littéraires, son attachement $\mathrm{au}$ « format livre », son caractère intimiste et sa recherche d'un nouveau lectorat. Cependant, la critique continue d'appliquer les outils d'analyse empruntés à la bande dessinée sans développer de théorie à même de prendre en compte l'ambiguïté du média « roman graphique », qui oscille entre bande dessinée, album, carnet de voyage, journal intime, carnet de croquis et se dilue dans ses influences photographiques, théâtrales, cinématographiques, architecturales, etc. ${ }^{32}$.

Un propos similaire peut tout à fait s'appliquer aux webséries : c'est en 2005 que le phénomène commence à s'imposer en Espagne, avec la série Qué vida más triste $^{33}$. Depuis, des formes variées se sont développées, explorant les limites, les emprunts possibles, les frictions entre divers médias, de la série télévisée à la cuisine, en passant par le cinéma, le théâtre, le documentaire, etc. Comment, dès lors, se saisir d'un objet aussi mouvant ? Le fait que la plupart des monographies l'abordent depuis les sciences de la communication semble témoigner de la difficulté à l'identifier, à l'explorer en tant que production culturelle et/ou création artistique.

Pour se saisir de ces objets depuis la perspective qui est la nôtre, celle de la création et des arts, l'intermédialité s'est donc imposée. De fait, la critique intermédiale permet d'appréhender pertinemment cette instabilité médiatique, soit

croisements intermédiatiques — voire intermédiaux, pour certaines webséries - mais elle est également au cour du questionnement, d'une part, sur l'influence que le milieu dans lequel émerge et s'inscrit un média va exercer sur celui-ci et, d'autre part, sur la naissance d'une communauté autour du média en question.

${ }^{32}$ Le roman graphique galicien Ardalén de Miguelanxo Prado reflète la capacité du média à intégrer et être influencé par d'autres médias extrêmement diversifiés. Pour une étude approfondie des relations intermédiales dans cette ouvre, voir l'article suivant: Agatha Mohring, « Dé-territorialisations, flous identitaires et frontières intermédiales dans le roman graphique galicien Ardalén de Miguelanxo Prado », Litter@ Incognita, Toulouse : Université Toulouse-Jean Jaurès, $\mathrm{n}^{\circ} 7, \quad$ 2016, s.p. http://blogs.univ-tlse2.fr/littera-incognita2/2016/10/03/de-territorialisations-flous-identitaires-et-frontieres-intermediales-dans-leroman-graphique-galicien-ardalen-de-miguelanxo-prado/ (consultation le 7 juin 2017).

33 Websérie de Natxodel Agua et Rubén Ontiveros, 2005-20ro. Pour une analyse plus détaillée de l'émergence et du fonctionnement intermédial de cette websérie, voir l'article suivant : Agnès Surbezy, «Internet en series de Qué vida más triste a Descarga completa », Letral, $\mathrm{n}^{\circ} \mathrm{II}$, décembre 2013, p. I27-134. La websérie est consultable en ligne à l'adresse: http://Quevidamastriste.com (consultation le I8 avril 20I7). 
en proposant de nouvelles modélisations, soit en réarticulant des concepts préexistants, issus d'autres disciplines et méthodologies.

Ainsi, le recours aux études intermédiales nous a-t-il permis d'étayer notre réflexion sur le statut médiatique du roman graphique et de la websérie. Les classifications telles que celle proposée par André Gaudreault et Philippe Marion $\mathrm{d}^{\prime}$ « une gradation à trois temps $[\ldots]:$ apparition, émergence et avènement ${ }^{34}$ » ou celle des « trois procédés : la citation, l'exploitation et la séparation 35 », développée par Rick Altman, permettent d'établir le degré d'indépendance du roman graphique par rapport à la bande dessinée, ou de la websérie par rapport à la télévision ou au cinéma.

On peut donc établir que le roman graphique n'a pas encore atteint « l'avènement » mais est en voie de « séparation ${ }^{36}$ ». Quant aux webséries, la place croissante qui leur est accordée dans les festivals, l'intérêt avéré d'un public sensibilisé, la création de structures de production dédiées, etc., sont autant de signes qui nous amènent à penser, après avoir interrogé le stade d'émergence de la websérie, qu'il s'agit d'un média en cours d' « avènement », sinon advenu.

Lorsque l'on choisit de centrer l'étude sur des œuvres en particulier, qu'il s'agisse de webséries ou de romans graphiques, l'analyse des relations intermédiales qui les sous-tendent s'articule à différents niveaux, que l'on peut organiser à partir de la division micro/méso/macro. Le niveau micro-intermédial permet d'étudier l'intégration d'un média ou d'un procédé médiatique hétérogène en un point donné. Par exemple, dans le roman graphique En segundo plano de Josep Busquet, Pedro J. Colombo et Aintzane Lane (2015), un appareil photo numérique fait écran entre les personnages photographiés et le photographe, et certaines cases rappellent le contour d'un objectif photographique, créant ainsi une rupture médiatique ${ }^{37}$.

34 Gaudreault, Marion, 2006.

35 Rick Altman, «De l'intermédialité au multimédia : cinéma, médias, avènement du son », Cinémas, n IOI, 1999, p. 37-53, http://dx.doi.org/10.7202/024802ar (consultation le 7 juin 2017).

${ }^{36}$ Pour un développement plus approfondi sur le statut médiatique du roman graphique, voir l'article suivant : Agatha Mohring, « Le roman graphique contemporain, émergence d'un nouveau médium ou évolution intermédiale de la bande dessinée ? », L'intermédialité à la croisée des chemins: bilans et perspectives, Peter Lang, à paraître.

37 Pour une étude approfondie des relations intermédiales et des concepts d'immediacy et d'hypermediacy dans les romans graphiques En segundo plano et Fallos de raccord, voir l'article suivant: Agatha Mohring, «Les relations intermédiales entre immediacy et hypermediacy dans les bandes dessinées En segundo plano et Fallos de raccord: explorations et redéfinitions 


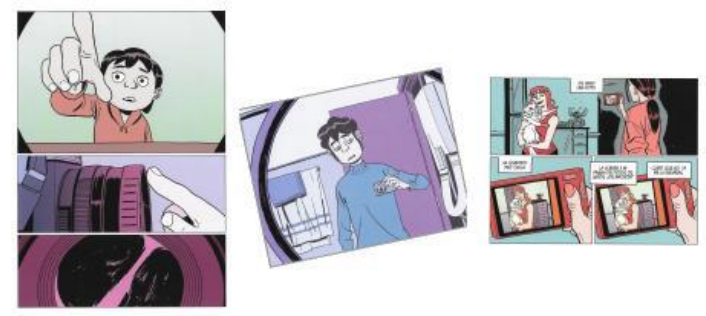

Figure 4. L'appareil photographique crée une rupture micro-intermédiale dans En segundo plano, Josep Busquet, Pedro J. Colombo et Aintzane Lane, 20I5, Madrid, Diábolo Ediciones. notions de transparence (immediacy) ou d'opacité (bypermediacy) médiatiques exposées par Jay David Bolter et Richard Grusin ${ }^{38}$ permet d'analyser le régime de visibilité et les tensions intermédiales, ainsi que le degré d'intégration du média extérieur à la websérie ${ }^{39}$ ou au roman graphique.

À l'échelle d'un épisode, ou d'un ou plusieurs chapitres d'un roman graphique, se tissent des relations méso-intermédiales qui sous-tendent l'organisation de l'œuvre à travers la présence d'un média ou d'un procédé médiatique étranger à la websérie ou au roman graphique. Dans Duelo de caracoles de Sonia Pulido et Pere Joan (20IO), les nombreuses coupes anatomiques et le discours médical sans cesse subverti reconfigurent la mise en page et l'organisation de l'œuvre pour proposer au lecteur un voyage intracorporel intimiste ${ }^{40}$.

du médium », Vincent Marie (dir.), BD in extenso, Paris, Les Manuscrits, coll. « Graphein », à paraître.

${ }^{38}$ Bolter, Grusin, 1999, p. 17.

39 Pour voir des exemples d'analyse de ce type de relations « micro-intermédiales » à l'œuvre dans les webséries, voir les articles suivants : Surbezy, 2013, p. I27-I34 et Agnès Surbezy, «Las webseries: intermedialidad y multipantallas », dans Florenchie, Breton, 2015, p. 179200 .

$4^{\circ}$ Pour une étude approfondie de l'intermédialité dans Duelo de caracoles, voir les articles suivants : Agatha Mohring, «Les coupes anatomiques dans le roman graphique Duelo de caracoles de Sonia Pulido et Pere Joan, entre dissection et réinvention de l'intériorité », dans Marie Garré Nicoara et Julie Postel (dir.), Corps béant, corps morcelé dans les arts scéniques et visuels, Louvain-la-Neuve, Éditions modulaires européennes, coll. « Arts, rites et théâtralité » (à paraître) et Agatha Mohring, «Lo íntimo y lo translúcido en la novela gráfica Duelo de caracoles de Sonia Pulido y Pere Joan », dans Marion Gautreau, Carmen Peña Ardid, Pascale 

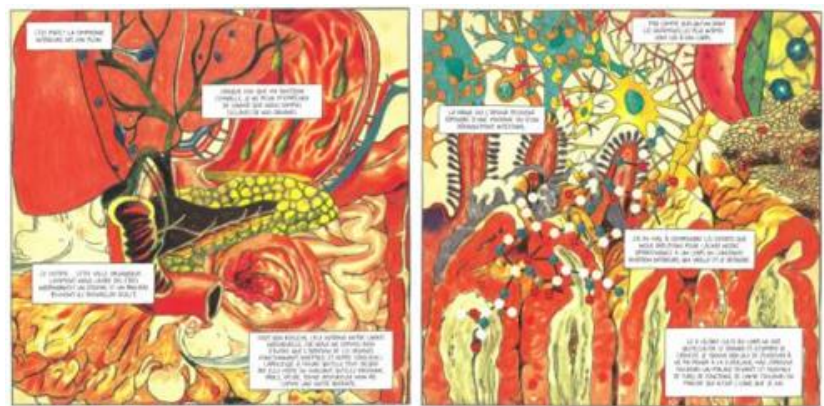

Figure 5. Coupe anatomique en pleine page dans Duelo de caracoles, Sonia Pulido et Pere Joan, 20Io, Madrid, Ediciones Sinsentido.

Un procédé assez comparable est à l'œuvre, par exemple, dans l'épisode « Los nunca para dummies » de la websérie colombienne Susana y Elvira ${ }^{4}$. Dans cet épisode - conformément à ce qu'annonce le titre — , font irruption des plans, mais aussi une séquence, reprenant visuellement la typographie et les codes couleur de la célèbre collection d'ouvrages For Dummies (Pour les nuls). Se trouvent également reproduits, dans les dialogues et la construction même de l'épisode, les procédés rhétoriques, le rythme, l'esprit de la collection. Ce croisement avec un phénomène éditorial international vient ainsi structurer l'épisode et le reconfigurer, entre production audiovisuelle, blogue de conseils et ouvrage de développement personnel, pour questionner nos sociétés et nourrir l'humour de la websérie.

Les relations macro-intermédiales, enfin, en ce qui a trait aux œuvres ellesmêmes, mettent en évidence une telle porosité qu'elles induisent fréquemment une réflexion sur les différents médias mis en jeu. De nombreux romans graphiques oscillent ainsi - d'un point de vue matériel, pragmatique, formel, conceptuel, éditorial et symbolique - entre roman graphique et carnet de croquis ( $S h b h$ de Nacho Casanova, 2015), ou entre journal intime et roman graphique (El hijo del legionario d'Aitor Saraiba, 20I5 et Los Juncos de Sandra Uve, 2006).

Peyraga et Sojo Kepa (dir.), L’image translucide dans les mondes hispaniques, Villeurbanne, Orbis Tertius, 2015, p. 25I-275.

${ }^{41}$ Websérie de María Fernanda Moreno et Marcela Peláez, 20I2-20I4. «Los nunca para dummies » est accessible en ligne : www.youtube.com/watch?v=ulwgvrCSs Nc (consultation le Io mars 20I7). 
Pour aborder ce niveau aussi, le jeu sur le degré de présence des médias évoqué plus haut est intéressant : si l'on prend l'exemple de la websérie Chicas del montón ${ }^{42}$, hommage explicite autant que parodique à l'univers almodovarien ${ }^{43}$, non seulement elle joue sur la relation avec le média cinéma, mais elle en exhibe une partie des ressorts, se plaçant plutôt dans un rapport d'bypermediacy, là même où le rapport au théâtre, média bien présent lui aussi, est moins exhibé, interrogeant la notion d'immediacy.
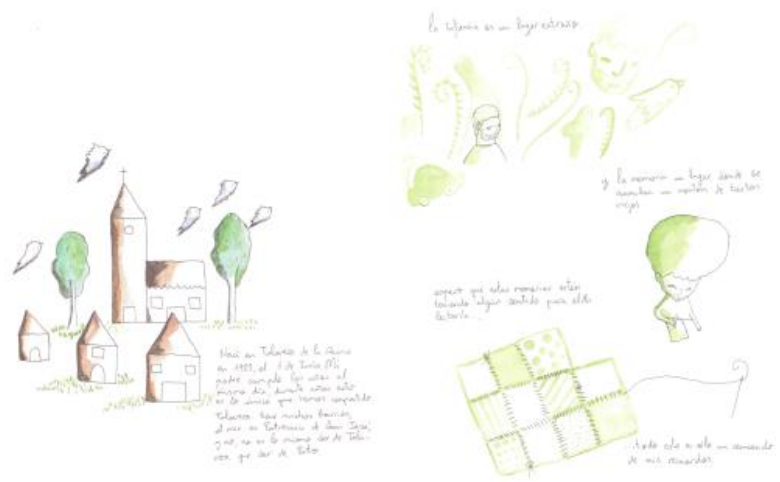

Figure 6. Macro-porosité intermédiale entre roman graphique, journal intime et carnet de croquis dans El hijo del legionario, Aitor Saraiba, 20I5.

S'ils sont à première vue différents, websérie et roman graphique ont donc en commun d'être des médias que l'on pourrait qualifier, à l'aune des travaux d'André Gaudreault sur le cinéma notamment, de médias non plus «émergents », mais « émergés ». L'intermédialité permet d'envisager leur complexité et leur richesse avec la souplesse et la profondeur de champ requises. De cette approche renouvelée des arts sont nés des concepts qu'une partie des chercheurs de l'équipe, qui développent des programmes de recherche orientés, ont transposés à des domaines d'application non artistiques (mais néanmoins liés à notre métier d'enseignantschercheurs) : la didactique des langues, et le congrès comme espace de formation.

42 Websérie de Fernando Gamero, 2013. La websérie est consultable en ligne: https://chicasdelmonton.wordpress.com/capitulos/ (consultation le 26 mars 2017).

${ }^{43} \mathrm{La}$ réflexion sur cette websérie et son rapport à l'intermédialité (et au transmédia) est actuellement développée, dans le cadre d'un cours de master d'études hispaniques, à l'Université Toulouse-Jean Jaurès. 


\subsection{L’INTERMÉdiALITÉ POUR ANALYSER, DEPUIS LES DISCIPLINES DE LA CRÉATION, DES OBJETS NON ARTISTIQUES}

L'intermédialité, en tant qu'approche, permet d'enrichir les travaux qui, depuis 2009, visent à mobiliser les théories et pratiques artistiques autour de projets d'innovation ${ }^{44}$. Dans le cadre du Laboratoire commun RiMeC (Réinventer le média congrès $)^{45}$ qui associe le laboratoire à l'entreprise Europa Organisation ${ }^{46}$, l'approche intermédiale ouvre de nouveaux angles d'analyse et apporte ainsi un éclairage pertinent à un objet de recherche singulier : le congrès ${ }^{47}$.

En proposant de s'intéresser non seulement aux médias eux-mêmes mais aussi aux relations entre les médias, l'intermédialité nous offre des outils d'analyse appropriés pour percevoir, décrire et analyser le réseau médiatique foisonnant que constitue le congrès (il s'agit ici d'un premier niveau d'analyse que l'on pourrait qualifier d'《intra-congrès »); pour envisager ce dernier comme un élément d'un système qui peut et doit être pensé au-delà des limites spatio-temporelles du seul événement (ce second niveau d'analyse replace le congrès dans une offre médiatique proposée par l'entreprise) et, enfin, pour l'inscrire dans un environnement médiatique riche et complexe (ce dernier niveau d'analyse dépasse le seul cadre pensé par l'entreprise).

Le corpus théorique issu des recherches intermédiales a permis de définir le congrès comme un média et d'en identifier ainsi quatre dimensions : un espace, une durée, des acteurs sociaux et des supports ${ }^{4}$. Dès lors, l'approche intermédiale a pu se

${ }^{44}$ Le laboratoire LLA-CREATIS porte une plateforme de recherche et de valorisation, la plateforme CRISO (Création et innovation sociétale) dont l'objectif est de mettre en œuvre, dans le domaine des arts, lettres et langues, des projets de recherche en lien avec des problématiques sociétales et des enjeux d'innovation, http://criso.univ-tlse2.fr (consultation le II mars 20I8).

45 Le Laboratoire commun RiMeC (Réinventer le média congrès) a été créé en 2013, grâce au soutien financier de l'Agence nationale de la recherche.

46 http://www.europa-organisation.com/fr/fr.html (consultation le ir mars 2or8).

${ }^{47}$ Nous précisons ici que les travaux du LabCom, de 2013-2016, ont porté principalement sur le congrès EuroPCR, congrès de cardiologie interventionnelle réunissant chaque année à Paris plus de $\mathrm{I} 2$ ooo professionnels du secteur (médecins, infirmier-e-s, technicien-ne-s, représentant-e-s des industries, etc.)

${ }^{48}$ Nous renvoyons à la définition du médium proposée par Éric Méchoulan : «Le médium est donc ce qui permet les échanges dans une certaine communauté à la fois comme dispositif sensible (pierre, parchemin, papier, écran cathodique sont des supports médiatiques) et comme milieu dans lequel les échanges ont lieu », dans Éric Méchoulan : «Intermédialités : le temps des illusions perdues », Intermédialités, $\mathrm{n}^{\circ} \mathrm{I}$, « Naître », 2003, p. 16. 
traduire en démarche critique, au cours des missions d'observation ${ }^{49}$, en orientant le regard spécifiquement sur ces quatre dimensions et sur leurs articulations. Elle a ainsi permis d'identifier, dans un premier temps, certaines problématiques susceptibles d'être explorées pour générer des innovations ${ }^{50}$. Ainsi, l'approche intermédiale a mis en lumière l'existence et le fonctionnement de «pré-sessions » et de «postsessions ». Si l’organisation du congrès s'articule largement autour de « sessions », pensées comme des médias autonomes, des espaces-temps émergent également, entre ces sessions, et deviennent, à leur tour, des formes médiatiques, non sans intérêt ni sans incidence sur les fonctions de formation et de socialisation assignées au congrès. Ces « pré-sessions » et « post-sessions » fonctionnent selon un régime spécifique basé principalement sur l'apparition de sous-espaces de communication interpersonnelle, sur l'absence des marqueurs de formalisme au bénéfice d'une dimension informelle des échanges, ou encore sur la présence d'objets intermédiaires fonctionnant comme lien entre les participants (une tasse à café, une feuille de papier, un smartphone personnel, etc.).

Ces observations de l'existant ont permis aux chercheurs, dans un second temps, de proposer une session innovante à l'entreprise et de l'expérimenter in vivos'. Cette session, dite « en trois temps », application directe des résultats de recherche, s'ouvre sur un premier temps visant à faire émerger les attentes et les besoins réels des participants. Pour ce faire, plusieurs sous-groupes sont constitués et prennent place dans un espace où les marqueurs de frontalité sont effacés au bénéfice d'une ambiance plus conviviale, propice aux échanges informels. Le deuxième temps est un temps de présentation, nourri du recueil des attentes et des besoins. Il se déroule dans un autre espace et renoue avec les codes classiques d'une session (formalisme des présentations, frontalité entre intervenants et participants, écran central avec diaporama, etc.). Enfin, le troisième temps invite les participants à dresser le bilan des présentations et à poser les questions qui resteraient en suspens. Il est marqué par le retour dans un espace moins formel, de manière à favoriser les échanges interpersonnels ${ }^{52}$.

${ }^{49}$ Missions d'observation menées lors de l'édition 2014 du congrès EuroPCR.

so Rémy Besson : «Une recherche intermédiale mise à l'épreuve du terrain. Retour sur la conception d'une session dite innovante $\gg$, Rapport de recherche, 20I5.

${ }^{51}$ L'expérimentation de la « Session en trois temps » a eu lieu lors de l'édition $2015 \mathrm{du}$ congrès EuroPCR.

${ }_{52} \mathrm{La}$ session en trois temps se fonde sur plusieurs principes : I) l'articulation entre deux temps de conversation et un temps de présentation; 2) l'articulation entre un espace informel (conversation) et un espace formel (présentation); 3 ) la conservation des codes classiques pour 
Cette session prétend intégrer à l'intérieur de la session le régime médiatique des « pré-sessions » et « post-sessions » (niveau I) et se veut, par conséquent, en adéquation avec les usages constatés. Fruit de l'application d'une recherche intermédiale, elle constitue un format nouveau, susceptible d'être développé et commercialisé par l'entreprise.

$\mathrm{Au}$ sein du LabCom, l'intermédialité permet également d'inscrire l'événement congrès dans un système médiatique plus vaste, composé d'autres événements, de ressources numériques, de publications, etc., concourant à la visée de formation des professionnels concernés. Les notions de convergence, de friction, de migration ou encore de transmédia semblent tout à fait opérantes pour caractériser les relations que le média congrès entretient avec les autres médias qui l'environnent. Cette approche «systémique » a pris la forme d'une «carte médiatique » permettant à l'entreprise de mieux penser la cohérence de son offre de services (niveau 2) mais aussi de mieux déterminer son positionnement vis-à-vis de la concurrence (niveau 3).

La portée intégratrice de l'approche intermédiale nous est apparue tout aussi intéressante pour la didactique des langues. Lors de la Journée d'études « Mettre en scène l'intermédialité : une approche de La Grieta de Gracia Morales et Juan Alberto Salvatierra 53 ", organisée par le laboratoire LLA-CREATIS en février 20I7, l'enseignement d'une langue étrangère a été analysé depuis une perspective intermédiale ${ }^{54}$. Cela a mis en évidence le réseau de médiations et d'interactions médiatiques qui peut sous-tendre le cours de langue et la constitution du « cours » lui-même comme un milieu construit sur des relations complexes et mouvantes entre

le temps de présentation; 4) la composition de sous-espaces de communication interpersonnelle (îlots) pendant le temps de conversation; 5) l'effacement des éléments de la frontalité scène/salle dans l'espace informel; 6) l'absence d'écran central dans l'espace informel; 7) la présence de plusieurs paper board dans l'espace informel; 8) la présence d'objets intermédiaires dans l'espace informel (café, lunch, paper board, etc.).

53 « Poner en escena la intermedialidad: un acercamiento a La Grieta de Gracias Morales y Juan Alberto Salvatierra », Journée d'études, Université Toulouse-Jean Jaurès, 22 février 2017, http://lla-creatis.univ-tlse2.fr/accueil/manifestations-scientifiques/mettre-en-scene-1intermedialite-une-approche-de-la-grieta--490436.kjsp?RH=I270I25529372.

54 Lumière, Émilie, «Teatro e intermedialidad en clase de idiomas: El proyecto $\mathrm{La}$ Grieta $\gg$ (communication sans actes), 22 février 2017. 
divers médias, parfois eux-mêmes intermédiatiques 55 . Cette étude du phénomène $\mathrm{du}$ cours de langue étrangère s'appuyait sur une expérience pédagogique pluri- et intermédiatique menée durant le second semestre 2016-2017 dans le cadre d'un enseignement d'espagnol pour l'acquisition du niveau $B_{2}{ }^{56}$. Cette expérience s'articulait sur une séquence consacrée au projet artistique La Grieta (websérie et pièce de théâtre, 201657), de la compagnie andalouse Remiendo Teatro, conçue pour un public hétérogène d'étudiants de licence et de master spécialistes d'autres disciplines que les langues (arts, psychologie, sociologie, etc.). La nature intermédiatique de cette séquence tenait aux activités proposées aux étudiants, qui croisaient des médias de différentes natures, pour certains interdépendants. À partir d'un dossier mêlant textes, images, documents audiovisuels et ressources en ligne (espace en ligne associé au cours et Internet), les étudiants ont visionné et analysé un chapitre de la websérie La Grieta et un extrait du spectacle La Grieta. Entre animales salvajes (captation vidéo réalisée par la troupe). Ils ont été amenés à réfléchir sur les contraintes institutionnelles et matérielles de la création de la websérie et du spectacle, ont réalisé des lectures dramatiques en classe et ont composé, en groupes, des fins alternatives à la pièce qu'ils ont mises en scène devant leurs camarades (certains de ces textes ont été envoyés à l'un des deux auteurs de la pièce). Cette séquence, menée sur trois séances de deux heures, avait pour but, dans une recherche d'authenticité linguistique et culturelle et de décloisonnement pédagogique, d'amener les étudiants à comprendre les modalités de création du projet artistique et à l'investir comme lecteurs, acteurs, apprentis auteurs, mais aussi comme spectateurs puisqu'ils ont assisté, dans le cadre du festival Universcènes de l'Université Toulouse-Jean Jaurès, à la représentation de la pièce en langue originale, surtitrée en français et interprétée en langue des signes française ${ }^{58}$. L'analyse de ce cours d'espagnol a montré l'intérêt pédagogique, social et même politique d'un enseignement intermédiatique, conçu depuis une intention intermédiale. Cela est d'autant plus évident dans le cas d'un

\$5 « Médias » entendus ici dans un sens large : textes, images, documents audio, vidéos, documents multimédias, médias physiques ou virtuels, espace de classe physique ou virtuel (espace numérique de travail - ENT), Internet.

${ }^{56}$ Niveau du Cadre européen commun de référence pour les langues, correspondant à un « utilisateur indépendant ».

57 Écrites et réalisées par les auteurs et membres de Remiendo Teatro, Gracia Morales et Juan Alberto Salvatierra, www.lagrieta.info/home (consultation le II mars 20I8).

${ }^{88} \mathrm{La}$ pièce a été jouée par la troupe universitaire «Barracrónicas », sous la direction d'Hegoa Garay, compagnie Les Anachroniques, www.anachroniques.fr/ (consultation le II mars 20I8). 
enseignement de langue et culture étrangères, car la démarche intermédiale permet que les étudiants travaillent assez naturellement plusieurs compétences communicationnelles 59 et se trouvent immergés plus facilement dans une réalité linguistique et culturelle spécifique. La perspective intermédiale, préalable à la conception du cours ou postérieure, dans un but analytique, incite à adopter une vision plurielle de la communication, de l'enseignement et de l'apprentissage, qui prenne en compte l'hétérogénéité des instances à l'origine des médiations, leur matérialité, le type de message transmis, leur(s) fonction(s) (pragmatique, linguistique, métalinguistique, culturelle, esthétique, politique, etc.), leur milieu et les lieux physiques ou virtuels (espaces universitaires ou extérieurs à l'université) pouvant prolonger et enrichir la pratique pédagogique non plus limitée à la seule salle de classe mais resituée dans son contexte ${ }^{60}$.

\section{ENRACINER / ARRAIGAR: QUELLE PLACE POUR L'INTENTIONNALITÉ INTERMÉDIALE DE L'ARTISTE ?}

I46 La critique littéraire et, de façon plus large, la critique en arts, est à la recherche de nouveaux modèles, de nouvelles théories, de nouveaux outils, pour (re)penser la création. Les réflexions qui ont précédé ont essayé de montrer comment l'intermédialité avait constitué, pour notre groupe, une façon d'enrichir, de renouveler notre regard, tenant davantage compte de l'environnement médiatique, culturel, social, dans lequel émergent les œuvres, et de la façon dont elles entrent en relation avec leur public.

Mais, à ce stade, nous nous sommes demandé si l'adoption de ce point de vue plus « panoramique », moins centré sur l'œuvre, n'était pas d'une certaine façon un retour en arrière critique, une façon de renouer avec les approches « l'homme et l'œuvre » antérieures au structuralisme. L'emploi d'une terminologie nouvelle ne masquerait-il pas en réalité un mouvement de va-et-vient de la critique entre des périodes où l'on privilégie un regard rapproché sur les œuvres - au risque de les décortiquer à l'excès - et des périodes où le point de vue est plus distancié, où les études sont moins artistiques que culturelles —, quitte à échouer à saisir la spécificité de la création observée ?

59 Compréhension de l'écrit et de l'oral, expression écrite et orale, et interaction orale selon le Cadre européen commun de référence pour les langues.

${ }^{60}$ Le laboratoire LLA-CREATIS développera ces axes de recherche en coordonnant notamment, en 2019, un numéro de la revue Les Langues modernes consacré au croisement entre intermédialité et didactique des langues. 
De fait, il faut reconnaître que, dans les études intermédiales, l'attention accrue portée à la matérialité du média, aux processus de médiation, aux conditions techniques ou technologiques d'émergence des médias, s'accompagne d'un effacement progressif de la frontière entre médias de communication et ce que nous avons choisi d'appeler les «médias artistiques ». Un nivellement s'opère dans le discours critique, qui semble réintroduire le geste artistique dans le tissu des actions humaines, sans le considérer comme ontologiquement différent.

William John Thomas Mitchell revendique ainsi, dans le cadre de l'émergence des visual studies, de pouvoir parler de cartes de vœux et de paquets de cigarettes tout autant que de cinéma ou de peinture ${ }^{\mathrm{GI}}$. Et pour accompagner le caractère protéiforme, mouvant de son «objet», il invoque la nécessité de dédisciplinariser, voire $\mathrm{d}^{\prime} \ll$ indisciplinariser $^{62} »$ les approches.

Henry Jenkins fonde, quant à lui, sa réflexion sur la «culture de la convergence » en s'appuyant exclusivement sur des événements et des phénomènes médiatiques populaires (Star Wars, Matrix, Harry Potter, etc.) dont il ne s'agit pas de questionner la valeur artistique, mais plutôt de voir comment ces produits sont symptomatiques d'un changement culturel dans lequel le spectateur-consommateur joue un rôle clé. Le rapport à la culture est exclusivement pensé comme un rapport de force entre les entreprises détenant les médias et les utilisateurs de ces médias. Le seul espace laissé aux « créateurs » - on ne parle pas d'artistes - ne déroge pas à cette logique :

Ceux qui font les médias ne pourront résoudre leur crise actuelle qu'en renégociant leur relation avec leurs utilisateurs. Le public à qui on a donné le pouvoir grâce à ces nouvelles technologies et qui occupe désormais un espace à l'intersection des anciens et des nouveaux médias exige de participer à cette culture. Les créateurs qui ne parviendront pas à aller au rythme de cette nouvelle culture participante verront leur public et leurs bénéfices décliner ${ }^{63}$. [Nous soulignons.]

${ }^{6}$ William John Thomas Mitchell, Iconologie: image, texte, idéologie, Paris, Les Prairies ordinaires, coll. «Penser/croiser », 2009, p. I3.

${ }^{62}$ Ibid.

${ }^{63}$ Jenkins, 20I3, p. 43. 
L'un des avantages d'une telle approche est de mettre davantage en lumière la façon dont l'œuvre se construit en fonction, à côté ou contre les autres médias qui l'environnent. Ainsi, Hervé Tullet, dans l'album pour enfants Un livre ${ }^{64}$, convoquet-il les gestes de la communication tactile sur tablette ou smartphone. Secouer, appuyer, faire glisser, etc. : chaque geste du lecteur transforme, à la page suivante, la couleur, la forme, le nombre ou l'ordre des figures géométriques représentées. Ce faisant, Hervé Tullet montre à quel point le mode de communication dynamicotactile du jeune enfant a servi de source d'inspiration pour penser une utilisation intuitive de ces interfaces relevant d'une technologie extrêmement complexe. Mais le propos d'Hervé Tullet consiste aussi à repoétiser avec humour, dans un livre de papier, ces gestes quotidiens, en les déconnectant de leur utilité directe ${ }^{65}$, en leur donnant le pouvoir de provoquer surprise et émotions.

Ce changement de perspective sur les productions médiatiques a conduit certains chercheurs, notamment au CRIalt, à définir l'intermédialité comme une approche, une interdiscipline, un «axe de pertinence ${ }^{66} »$, un ensemble d'outils critiques en construction. L'intermédialité consiste en une mise en relation, en une étude des relations entre l'objet et son environnement, en un refus de considérer les œuvres de façon insulaire ${ }^{67}$. Mais elle n'est pas considérée comme une qualité possible des œuvres elles-mêmes. Rémy Besson dans son article de synthèse écrit que « l'intermédialité n'est [...] pas pensée comme une propriété relative à un objet, mais comme correspondant à un changement de perspective de la part du chercheur/de celui qui observe le monde ${ }^{68} »$. Il ajoute en note qu' « il n'est pas possible de dire qu'un film, une série télévisée ou un site internet est (ontologiquement) intermédial. Il est, par contre, envisageable de concevoir/d'analyser une production culturelle en

\footnotetext{
${ }^{64}$ Hervé Tullet, Un livre, Paris, Bayard, 2010. Le trailer de l'album dans sa version anglaise est disponible: www.youtube.com/watch?v=Kj8IKCGm64\&index=3\&list=PLKhvAzfyr9stxyzaBHf-09V56COeI73EW (consultation le II mars 20I8).

6s Euriell Gobbé-Mévellec, « De l'album jeunesse aux applis livre : nouveaux dispositifs de lecture », Fanny Barnabé et Björn-Olav Dozo(dir.), Mémoires du livre/Studies in Book Culture, « Livre et jeu vidéo », vol. $5, \mathrm{n}^{\circ} 2$, printemps 2014.

${ }^{66}$ Jürgen E. Müller, «L'intermédialité, une nouvelle approche interdisciplinaire: perspectives théoriques et pratiques à l'exemple de la vision de la télévision », Cinéma et intermédialité, vol. Io, $\mathrm{n}^{\circ \mathrm{s}} 2-3$, printemps 2000, www.erudit.org/fr/revues/cine/2000-vio-n23-ciner88I/024818ar.pdf (consultation le 8 mars 2017).

${ }^{67}$ Albera, Boillat, Carou et Le Forestier, 2009.

${ }^{68}$ Besson, 2014.
} 
adoptant une approche intermédiale ${ }^{69} \gg$. Même s'il précise que « ce dernier point fait débat au sein de la communauté scientifique ${ }^{70} \gg$, c'est là une position assez marquée au CRIalt, ainsi que le revendique Philippe Despoix, dans son introduction au $\mathrm{n}^{\circ} 20$ de la revue Intermédialités:

Depuis ses débuts, la revue s'est fixé d'aborder l'intermédialité non comme un objet mais comme un réseau complexe de relations, matérielles, techniques, sociales et sémiologiques, permettant de dessiner a posteriori des formes de médiation culturelle de fait toujours hybrides ${ }^{71}$.

Et c'est peut-être là que le nivellement entre médias de communication et médias artistiques peut perdre, selon nous, de son efficacité. Il est difficile, en effet, depuis ce point de vue, de parvenir à montrer que le propos d'un artiste peut précisément consister à penser sa relation aux médias qui l'environnent.

Le propos - et la difficulté - est en réalité ici de reconnaître - et peut-être de légitimer - l'intention artistique, celle-là même que la tradition plaçait en amont de la confrontation avec la matière (plastique, sonore, verbale, etc.) et que l'ère contemporaine semble ramener au présent du geste créateur, dans une sorte de délégation aux appareils techniques qui président à notre société hypermédiatique. Dès lors, l'intention de l'artiste se trouve-t-elle liquidée, anéantie par les forces plurielles du réseau médiatique et la présence toujours plus importante du récepteur à l'intérieur de son œuvre ? Ou bien est-elle simplement déplacée vers l'aval, gardant alors une existence propre que le récepteur pourrait encore identifier, alors même qu'il intervient toujours plus lui-même comme cocréateur des dispositifs artistiques ? Michel Guérin, pour sa part, continue de souligner la place à part de l'art immergé dans un tissu de médialités en tous genres :

L'activité artistique est seulement l'une des branches de ce nouvel arbre de la connaissance d'un genre particulier, et, au-delà du multimédia (c'est-à-dire d'une hybridation de techniques) et de l'inter- ou transmédialité (c'est-à-dire de passages de médiums les uns dans les autres, interactions ou

\section{Ibid.}

70 Ibid.

${ }^{71}$ Philippe Despoix, «Traverser dix ans d'Intermédialités», Intermédialités, «Traverser/Crossing $», \mathrm{n}^{\circ}$ 20, printemps 20I2-automne $2013, \mathrm{p} .7$. 
transpositions), c'est le modèle d'une société en réseau qui s'impose partout $^{72}$.

En ce sens, dans la complexité des événements médiatiques actuels, comment savoir où se situe l'intentionnalité d'un geste artistique ? À cette question, on pourra répondre de deux manières, qui se font écho.

D'une part, la figure de l'artiste continue d'être le dépositaire de « l'équipement » (entendu par Walter Benjamin comme à la fois la technique et la culture ${ }^{73}$ ) propre à son époque. Qu'il traduise ou qu'il éprouve les éléments constitutifs de son environnement (social, politique, moral, etc.), le créateur contemporain peut difficilement échapper à une pensée critique par et sur les médias qui imprègnent si fortement son environnement ${ }^{74}$. Il y a donc fort à parier que son œuvre (ou ses propositions artistiques) soit à recevoir comme un questionnement sur l'ère médiatique et les phénomènes de médiation, à part entière ou de façon périphérique. Il en va ainsi des œuvres qui mettent explicitement le médiatique en leur centre (thème) ${ }^{75}$, ou de celles qui mettent en auvre une médiativité complexe dans une sorte de mise en abyme à effet critique ${ }^{76}$, ou encore de celles qui épousent par analogie les codes médiatiques pour une (re)poétisation du média choisi.

${ }^{72}$ Michel Guérin, «Qu'est-ce qu'un médium artistique ?», Appareil, 2016, n 17, http://appareil.revues.org/2308 (consultation le I5 mai 20I7).

73 Walter Benjamin, «L'œuvre d'art à l'époque de sa reproduction mécanisée », Écrits français, Paris, Gallimard, coll. « Bibliothèque des idées », I99I.

74 « Les artistes actuels échappent d'autant moins au multimédia, à la transmédialité ou à l'intermédialité, que les y incitent, depuis l'invention de la photographie, les appareils, euxmêmes aujourd'hui déterminés par les technologies de l'information et de la communication et, au-delà, par l'omniprésence des réseaux dans la réalité aussi bien que dans les têtes. À l'ère de la network society, une pratique artistique peut-elle se limiter à un seul médium ? », Guérin, 20I6, p. I.

75 Voir, par exemple, les travaux d'Emmanuelle Garnier : « Terrorismo, intermedialidad y omnisciencia: Boris Godunov, un espectáculo de La Fura del Baus (2008) », Letral, no II, sección: Estudios transatlánticos, diciembre 20I3, p. I-IO, www.proyectoletral.es/revista/index.php?id_num=I3 (consultation le II mars 20I8); « De los dos lados de la webcam: Venecia, una performance de Angélica Liddell », José Romera Castillo (dir.), Teatro e Internet, Madrid, UNED, 2013, p. 403-419; « La fábula y la técnica: un acercamiento intermedial al "lenguaje furero" (La cuna de una nación, 2012) », Séminaire international «Performa (I) », I6-I8 novembre 2016, Universidad Carlos III de Madrid, à paraître.

${ }^{76}$ Parmi les travaux développés par l'équipe toulousaine en ce sens, citons : Émilie Lumière, «Intermédialité et mise en scène de l'histoire au théâtre : Retrato de gran almirante 
D'autre part, à l'ère du transhumanisme, voire du post-humanisme, où la technologie conduit à la prégnance de l'artificiel sur le naturel, revient en force la question des sources archaïques de l'art, qui lient celui-ci au corps, aux pulsions, aux sens et aux émotions, là où la technicité autonomisée ne proposerait que froideur programmatique, loin de toute intention artistique. En ce sens, la tentation est grande de revenir aux sources de l'intentionnalité artistique, qui assume une certaine opacité de la genèse créatrice, son caractère fondamentalement énigmatique.

Ainsi, une œuvre d'art est-elle située au carrefour d'une intention naturelle en partie impénétrable et d'une intention choisie, qui met en perspective critique certains éléments du monde dans lequel elle fait irruption et, en particulier, les médias eux-mêmes, qui, tout à la fois, la constituent dans son environnement et font l'objet de son discours critique. En ce sens, une ouvre artistique (qu'elle soit objet, geste, événement, etc.) peut être envisagée comme intrinsèquement intermédiale, car douée, pour ainsi dire, de la conscience d'être un média réfléchissant.

\section{CONCLUSION ET PERSPECTIVES}

Ce parcours rapide des différentes orientations qu'ont pu prendre les travaux de notre groupe au fil des dernières années ${ }^{77}$ montre combien le défi — un peu fou d'embrasser l'intermédialité est venu bousculer de façon féconde nos travaux, renforçant l'opérabilité critique du dispositif artistique en l'ouvrant aux médias dans leurs multiples dimensions (inscriptions, supports, milieux), invitant à un recul historique nécessaire à l'heure de comprendre les grands changements sociétaux

con perros de Luis Riaza », à paraître; Monique Martinez Thomas, « ¿A qué jugamos? », Primer Acto, $\mathrm{n}^{\circ}$ 352, I/2017, p. 270-273; Agatha Mohring y Monique Martinez Thomas, « Jeux intermédiaux dans la pièce $\mathrm{La}$ Grieta, entre animales salvajes et ses satellites vidéos » (inédit); Agatha Mohring, «La Grieta, entre animales salvajes, satellite d'un projet intermédial », introduction à Gracia Morales et Juan Alberto Salvatierra, La grieta, entre animales salvajes / La Fissure, tête à tête avec des bêtes sauvages, trad. de l'atelier de Mı du CETIM sous la direction de Monique Martinez Thomas, Toulouse, Presses universitaires du Midi, 20I7, p. 9-27; et plus généralement, le programme de la journée d'étude du 22 février 2017 sur « Mettre en scène l'intermédialité : une approche de La Grieta », www.univ-tlsez.fr/accueil/agenda/mettre-enscene-l-intermedialite-une-approche-de-la-grieta--490436.kjsp (consultation le II mars 20I8).

77 La bibliographie des travaux réalisés dans le cadre du séminaire Intermedialidades est téléchargeable sur le site de LLA-CREATIS: http://lla-creatis.univtlse2.fr/accueil/programmes-de-recherche-20I5-2020-/axe-i-dispositifs-artistiques-creation-etintermedialite/dispositifs-artistiques-intermedialite-transmedialite/programme-dispositifsartistiques-intermedialite-transmedialite-370540.kjsp? RH=Axes_llacreatis 
induits, entre autres, par la révolution numérique, et révélant une dimension politique de façon plus directe. Ce déport vers les champs des sciences sociales a permis, parallèlement, de faire un retour sur l'art et de (re)questionner la spécificité du média artistique, dont les modalités d'émergence répondent à un processus intentionnel doué d'une capacité de questionnement pour ainsi dire métamédiatique à travers des œuvres, des actions, des événements concrets. Dès lors, la place du média artistique dans le vaste maillage intermédiatique dans lequel tout un chacun est désormais pris pourrait s'envisager comme étant à part, distincte; elle est surplombante, « intel-ligente » car l'art sait et permet de faire retour sur l'humain.

Cette vision nous a séduits. Il reste à l'éprouver solidement ${ }^{78}$. Les deux années qui viennent seront dédiées à étayer cette hypothèse. Au risque - passionnant - de la voir battue en brèche à l'occasion de débats que nous appelons de nos voux, particulièrement avec les coauteurs du présent numéro consacré à la cartographie actuelle des travaux sur l'intermédialité.

${ }^{78}$ Nous nous attelons notamment à la constitution d'un lexique des mots de l'intermédialité pour l'étude des médias artistiques. 


\title{
De la critique des dispositifs à l'intermédialité pour approcher les productions artistiques : bilan des travaux du séminaire Intermedialidades (Université Toulouse-Jean Jaurès, France)
}

\author{
LLA-CREATIS, UNIVERSITÉ TOULOUSE-JEAN JAURĖS
}

\section{RÉSUMÉ}

À l'occasion de la publication du numéro « Cartographier/Mapping » de la revue Intermédialités, le groupe des hispanistes du laboratoire LLA-CREATIS de l'Université Toulouse-Jean Jaurès a souhaité établir un bilan des travaux qu'il a menés depuis quatre ans en lien avec l'intermédialité, au sein du séminaire Intermedialidades. L'article s'attache ainsi à dégager les principales lignes de force de ces travaux, notamment : l'articulation des outils de l'intermédialité avec ceux de la critique des dispositifs développée au sein du laboratoire ces dix dernières années; l'intérêt de l'intermédialité entendue comme une approche conceptuelle plurielle pour explorer « l'entre-jeu complexe des médias » (Müller) dans toute production culturelle - artistique ou non —, quelles que soient son ampleur et sa complexité, et en mesurer finement les multiples enjeux; l'hypothèse, enfin, que dans cette perspective très large, il soit possible d'affirmer une spécificité du média artistique, que l'analyse d'objets intermédiaux permet de percevoir dans la mesure où ces objets peuvent porter la trace d'une intention artistique intermédiale, réfléchissant précisément sur la porosité, l'articulation, la friction entre les médias et sur la place de la création dans l'espace médiatique où elle émerge.

\section{ABSTRACT}

The publication of this issue on "Cartographier/Mapping" provides an opportunity for Hispanist researchers at the LLA-CREATIS Laboratory (at the University of Toulouse) to reflect upon four years of research on intermediality conducted within the Intermedialidades seminar. This article presents their contributions on the linkages between intermedial tools and a decade-long research on "dispositive theory" at the LLA-CREATIS Laboratory. It stresses intermediality as a pluralistic approach that explores the "complex media midfield" (Müller) of cultural-artistic and nonartistic-products. Intermediality takes stock of the scope and sophistication of these products and provides a fine-grained analytical tool to address their multiple challenges. This article advances the hypothesis that by using this broad outlook one may outline what is specific in artistic media through the analysis of intermedial objects, under the condition that these artistic products display an intermedial artistic 
intent - in other words, that they reflect on the permeability, linkages, and frictions between various media, as well as on the media environment in which creation occurs.

\section{NOTE BIOGRAPHIQUE}

Fabrice Corrons, Emmanuelle Garnier, Euriell Gobbé-MÉvellec, Émilie Lumière, Monique Martinez Thomas, Agatha Mohring, Bruno Péran, Agnès Surbezy et AnNe-Claire Yemsi-Pailuissé sont membres du laboratoire LLA-CREATIS de l'Université Toulouse-Jean Jaurès et travaillent sur la création contemporaine hispanophone dans des champs variés (théâtre, performance, roman graphique, webséries, album pour la jeunesse, haute gastronomie, danse, traduction). À la suite de la publication de l'ouvrage collectif numérique Dispositivo y artes: una nueva berramienta critica para analizar las producciones contemporáneas (Naque, 20I4), l'équipe, réunie dans le cadre du séminaire Intermedialidades, a choisi de faire évoluer sa perspective critique depuis la critique des dispositifs vers l'intermédialité, notamment au contact de plusieurs chercheurs du CRIalt. 